TITLE:

\title{
Directional linearly polarized terahertz emission from argon clusters irradiated by noncollinear double-pulse beams
}

\section{AUTHOR(S):}

Mori, Kazuaki; Hashida, Masaki; Nagashima, Takeshi; Li, Dazhi; Teramoto, Kensuke; Nakamiya, Yoshihide; Inoue, Shunsuke; Sakabe, Shuji

\section{CITATION:}

Mori, Kazuaki ...[et al]. Directional linearly polarized terahertz emission from argon clusters irradiated by noncollinear double-pulse beams. Applied Physics Letters 2017, 114(24): 241107.

\section{ISSUE DATE:}

2017-12-11

URL:

http://hdl.handle.net/2433/267218

\section{RIGHT:}

(c) 2017 Author(s). Published by AIP Publishing.; This article may be downloaded for personal use only. Any other use requires prior permission of the author and AIP Publishing. This article appeared in Kazuaki Mori, Masaki Hashida, Takeshi Nagashima, Dazhi Li, Kensuke Teramoto, Yoshihide Nakamiya, Shunsuke Inoue, and Shuji Sakabe, "Directional linearly polarized terahertz emission from argon clusters irradiated by noncollinear double-pulse beams", Appl. Phys. Lett. 111, 241107 (2017) and may be found at https://doi.org/10.1063/1.4991736. 


\section{Directional linearly polarized terahertz emission from argon clusters irradiated by noncollinear double-pulse beams}

Cite as: Appl. Phys. Lett. 111, 241107 (2017); https://doi.org/10.1063/1.4991736

Submitted: 22 June 2017 • Accepted: 06 November 2017 • Published Online: 15 December 2017

(D) Kazuaki Mori, Masaki Hashida, Takeshi Nagashima, et al.
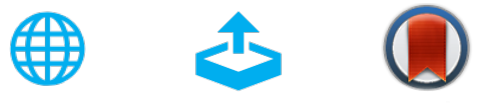

View Online

Export Citation

\section{ARTICLES YOU MAY BE INTERESTED IN}

Increased energy of THz waves from a cluster plasma by optimizing laser pulse duration AIP Advances 9, 015134 (2019); https://doi.org/10.1063/1.5075712

Intense terahertz emission from atomic cluster plasma produced by intense femtosecond laser pulses

Applied Physics Letters 99, 261503 (2011); https://doi.org/10.1063/1.3672814

Observation of broadband terahertz wave generation from liquid water

Applied Physics Letters 111, 071103 (2017); https://doi.org/10.1063/1.4990824

耳QBLOX

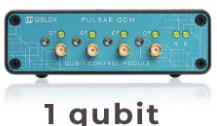

Shorten Setup Time Auto-Calibration More Qubits

\section{Fully-integrated} Quantum Control Stacks Ultrastable DC to $18.5 \mathrm{GHz}$ Synchronized $<<1$ ns Ultralow noise

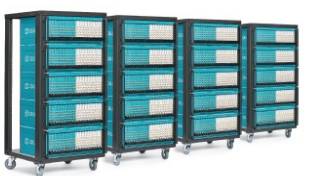

100s qubits

visit our website > 


\title{
Directional linearly polarized terahertz emission from argon clusters irradiated by noncollinear double-pulse beams
}

\author{
Kazuaki Mori, ${ }^{1,2}$ Masaki Hashida, ${ }^{1,2}$ Takeshi Nagashima, ${ }^{3}$ Dazhi Li, ${ }^{4}$ Kensuke Teramoto,,${ }^{1,2}$ \\ Yoshihide Nakamiya, ${ }^{1}$ Shunsuke Inoue, ${ }^{1,2}$ and Shuji Sakabe ${ }^{1,2}$ \\ ${ }^{1}$ Advanced Research Center for Beam Science, Institute for Chemical Research, Kyoto University, Gokasho, \\ Uji, Kyoto 611-0011, Japan \\ ${ }^{2}$ Department of Physics, Graduate School of Science, Kyoto University, Kitashirakawa, Sakyo, \\ Kyoto 606-8502, Japan \\ ${ }^{3}$ Faculty of Science and Engineering, Setsunan University, 17-8 Ikeda-Nakamachi, Neyagawa, \\ Osaka 572-8508, Japan \\ ${ }^{4}$ Institute for Laser Technology, 1-8-4 Utsubo-honmachi, Nishi-ku, Osaka 550-0004, Japan
}

(Received 22 June 2017; accepted 6 November 2017; published online 15 December 2017)

\begin{abstract}
It has been demonstrated that the interaction between argon clusters and intense femtosecond double laser pulses with appropriate intervals in time and space provides important properties for terahertz electromagnetic wave generation, namely, high forward directivity, power enhancement, and linear polarization with a variable direction. Irradiating argon clusters with double pulses ( 1 and $3 \mathrm{~mJ}, 40 \mathrm{fs}, 810 \mathrm{~nm}$ ) in 133-ps and 40- $\mu$ m intervals results in terahertz wave emission in the forward direction that is 10 times greater than that for a single pulse. The polarization direction of terahertz electromagnetic waves can be varied by changing the relative focal positions of the first and second pulses. Published by AIP Publishing. https://doi.org/10.1063/1.4991736
\end{abstract}

In the past few decades, the scientific and engineering applications of terahertz (THz) waves have advanced remarkably with the rapid progress of laser technology. Intense $\mathrm{THz}$ waves have attracted attention for various applications. ${ }^{1-5}$ Currently, 0.9-mJ THz wave pulses are generated by optical rectification in an organic nonlinear crystal. ${ }^{6}$ However, it is difficult to increase the $\mathrm{THz}$ wave energy with this method because the damage threshold of the nonlinear crystal limits the incident laser energy. Using a plasma as the THz-wavesource element would reduce this limitation considerably and is a promising method for generating intense $\mathrm{THz}$ waves. Laser plasmas produced from a solid target generate $0.7-\mathrm{mJ}$ $\mathrm{THz}$ waves by sheath proton acceleration. ${ }^{7}$ However, this method is not suitable for repeated generation of $\mathrm{THz}$ waves because of debris from the solid target and the difficulty in replenishing the target continuously. A gas target can overcome these problems but provides $\mathrm{THz}$ waves with much lower energies than those from a solid target. ${ }^{8,9}$ To have the benefits of both solid and gas targets, clusters consisting of atoms have been proposed as a target, ${ }^{10}$ and the generation of $\mathrm{THz}$ waves from cluster plasmas has been studied experimentally and theoretically. ${ }^{11-14}$ Jahangiri et al. reported that an atomic-cluster plasma generates 600 times more $\mathrm{THz}$ waves than a gas plasma does, ${ }^{11,12}$ thereby showing the potential of an atomic-cluster plasma as a THz wave source.

However, in conventional methods, $\mathrm{THz}$ waves from cluster plasmas produced by irradiation with a single-pulse laser beam generally have radial polarization and conical angular distribution. This is inconvenient for applications because such characteristics make handling the waves difficult and less efficient. Therefore, it is important to develop intense $\mathrm{THz}$ wave sources with good characteristics, in particular directional linearly polarized emission. For a gas target, the use of multiple incident laser pulses has been proposed to improve the radiation properties. Generating $\mathrm{THz}$ waves via two-color laser focusing in a gas has overcome the problematic radiation properties of $\mathrm{THz}$ waves from a gas plasma. ${ }^{15-17}$ In this scheme, linearly polarized $\mathrm{THz}$ waves are generated in the forward direction. This requires precise adjustment of the temporal deviation between the two pulses to match their phases. ${ }^{18}$ An additional challenge is the damage threshold of the second-harmonic-generation crystal, limiting the $\mathrm{THz}$ output. As a method using two single-color pulses, a bi-filamentation scheme has been proposed. ${ }^{19}$ Although $\mathrm{THz}$ wave generation in the forward direction has been measured, the power is much lower than that for solid or cluster targets.

In this study, we use a cluster target to generate the plasma and the $\mathrm{THz}$ waves separately with double-pulse beams to search for appropriate intervals in both time and space for the pulses to be focused into the plasma. THz waves are emitted with linear polarization, and their power is greatly enhanced in the direction of laser propagation.

A schematic diagram of the experimental setup is shown in Fig. 1. We use a Ti:sapphire chirped pulse amplification laser system operating at a central wavelength of $810 \mathrm{~nm}$. The laser pulse has a duration of 40 fs (FWHM). The laser beam is separated into two beams: the first pulse with an energy of $1 \mathrm{~mJ}$ and the second pulse with an energy of $3 \mathrm{~mJ}$. The pulse energies of the two pulses are measured separately in front of a glass chamber with a pyroelectric energy meter. The two pulses are linearly polarized with a polarization parallel to the optical table. The time delay between the two pulses is varied from 0 to 500 ps with 0.03 -fs resolution by a direct-drive translation stage (ANT95-100-L, Aerotech Inc.). The two beams are recombined and focused onto the argon clusters by a plano-convex lens with a focal length of $200 \mathrm{~mm}(\mathrm{~F}=10)$. The first and second laser beams can be focused in Gaussian spots with diameters of 12 and $15 \mu \mathrm{m}$ $\left(\mathrm{FWe}^{-1} \mathrm{M}\right)$, respectively. The focal position of the second 


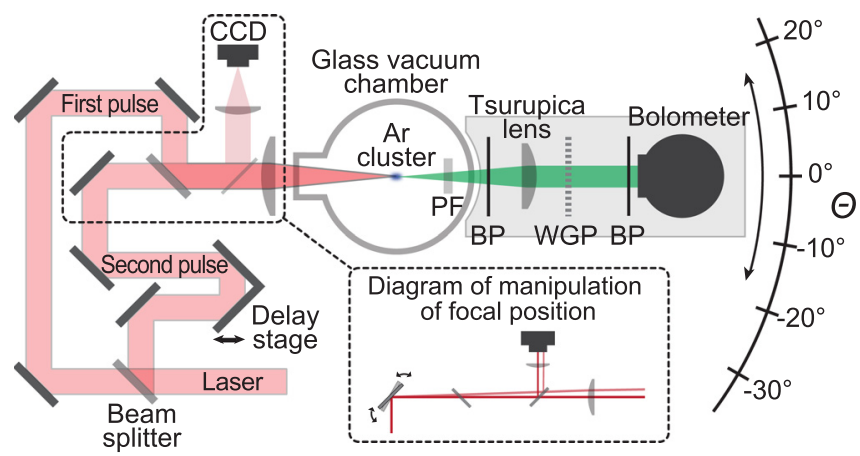

FIG. 1. Schematic diagram of the experimental setup. PF: polystyrene foam; BP: black polypropylene filter; WGP: wire-grid polarizer; and $\Theta$ : detection angle of $\mathrm{THz}$ waves.

pulse can be varied from 0 to $100 \mu \mathrm{m}$ (corresponding to $0-500 \mu \mathrm{rad}$ ) by a mirror just before the beam combiner. The focal position (pointing) of the two beams is monitored using a CCD camera during the experiment.

Argon clusters are produced in the center of a glass vacuum chamber by injecting argon gas with a backing pressure of $7 \mathrm{MPa}$ through a conical nozzle whose length is $20 \mathrm{~mm}$ and whose input and output diameters are 0.5 and $5 \mathrm{~mm}$, respectively. The distance of the nozzle from the focal point is $10 \mathrm{~mm}$. The typical radius of clusters is measured as $R_{0} \approx 5 \mathrm{~nm}$ by the Rayleigh scattering method, ${ }^{20}$ and the atomic density in the interaction region is estimated to be approximately $6 \times 10^{17} \mathrm{~cm}^{-3}$. The $\mathrm{THz}$ waves generated by irradiating the argon clusters with intense laser pulses are collimated by a Tsurupica lens (Pax Co.) and detected using a helium-cooled InSb bolometer (QFI-2BI, QMC Instruments Ltd.) whose detectable frequency range is up to approximately $2 \mathrm{THz}$. Five-millimeter-thick polystyrene foam and $60-\mu \mathrm{m}$-thick black polypropylene filters are placed in front of the bolometer to block residual laser pulses and undesirable emissions from the plasma. The detection system consists of the bolometer, a wire grid, the Tsurupica lens, and the black polypropylene filters on an optical plate (Fig. 1). To measure the angular distribution of the $\mathrm{THz}$ wave emission, the detection system is moved in an arc from $\Theta=80^{\circ}$ to $-60^{\circ}$ with a resolution of $4^{\circ}$, and a chamber made of fused silica glass with $90 \%$ transparency at $0.5 \mathrm{THz}$ is used. ${ }^{21}$ The polarization properties and spectrum of the $\mathrm{THz}$ waves are measured with wire-grid polarizers (extinction ratio approximately $10^{-5}$ at $0.5 \mathrm{THz}$ ) and a Martin-Puplett interferometer, respectively. Each data point is obtained by averaging 16 pulses.

First, the $\mathrm{THz}$ power dependence on the focal position of the second pulse relative to that of the first pulse is measured. Figure 2(a) shows the definition of the relative focal position of the double-pulse beams irradiating the argon clusters. The focal position of the second pulse relative to that of the first pulse is shown in Cartesian coordinates $(x, y)$ and cylindrical coordinates $(r, \theta)$. Figure 2 (b) shows the $\mathrm{THz}$ power dependence on the focal position of the second pulse relative to that of the first pulse at a delay time of $133 \mathrm{ps.}$ Although almost no $\mathrm{THz}$ waves are generated when the first and second pulses are irradiated collinearly [i.e., $(x, y)=(0$, $0)], \mathrm{THz}$ wave generation is enhanced when the two pulses are irradiated noncollinearly [i.e., $(x, y) \neq(0,0)]$ and is
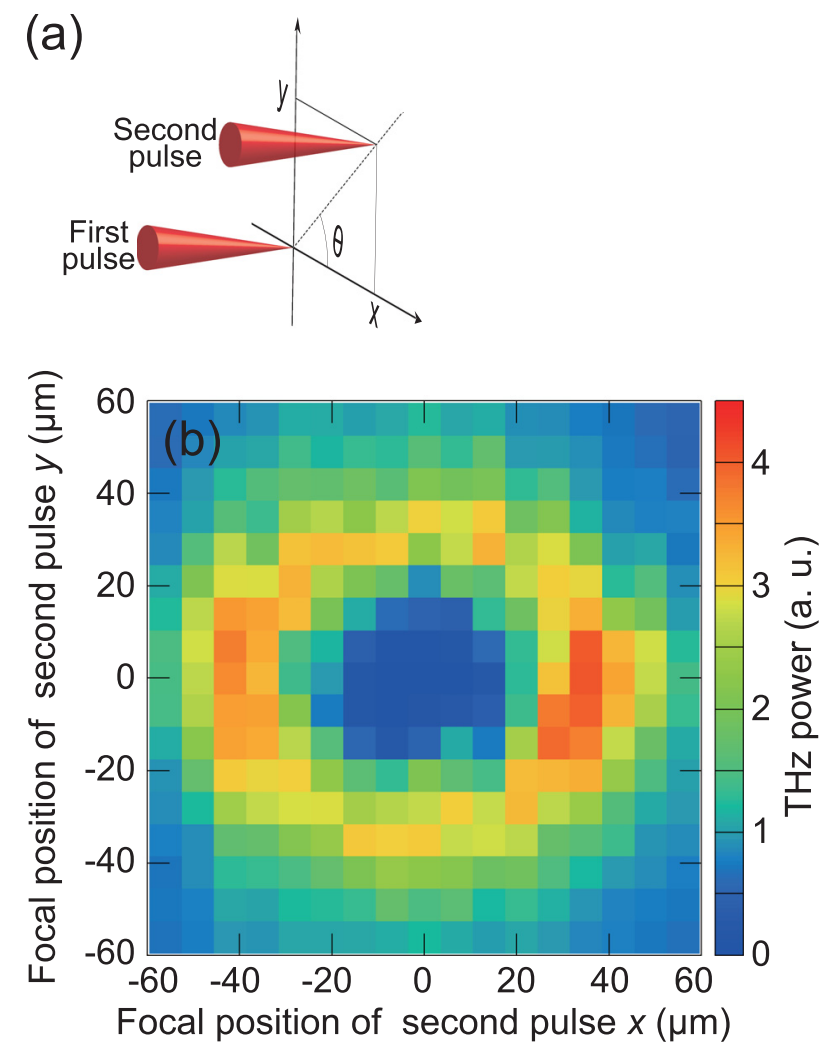

FIG. 2. (a) Schematic of $x, y$, and $\theta$ defined as the focal position of the second pulse relative to the focal position of the first pulse. (b) Dependence of THz power on the focal position $(x, y)$ of the second pulse. The delay time between the two pulses is $133 \mathrm{ps}$. THz power is detected in the laserpropagation direction $\left(\Theta=0^{\circ}\right.$ in Fig. 1).

maximized by shifting the second pulse by approximately $40 \mu \mathrm{m}$ regardless of $\theta$. In this experiment, THz waves are detected in the direction of $\Theta=0^{\circ}$. The $\mathrm{THz}$ waves are detected at a solid angle of $7.9 \times 10^{-3} \mathrm{sr}$, which is much larger than the maximum shift of the second pulse $(500$ $\mu \mathrm{rad})$. Therefore, the shift of the second pulse is small enough not to influence $\mathrm{THz}$ detection. Under conditions in which the delay time is $133 \mathrm{ps}$ and the focal position of the second pulse is $(x, y)=(0,40 \mu \mathrm{m})$, the THz-power stability (standard deviation/average value) is $11 \%$.

Figure 3(a) shows the dependence of $\mathrm{THz}$ power on the delay time of the second pulse. The focal position of the second pulse is shifted to $(x, 0)(\theta=0)$. As the delay time is increased from 1 to 500 ps, position $x$ of the second pulse that gives the maximum $\mathrm{THz}$ wave power increases, which is shown in Fig. 3(b) more clearly. Figure 3(b) shows the second-pulse positions that give each maximum $\mathrm{THz}$ power in Fig. 3(a) as a function of their delay time. The secondpulse position for maximum $\mathrm{THz}$ wave emission moves at a speed of $8 \times 10^{4} \mathrm{~m} / \mathrm{s}$.

Figure 4 shows the angular distribution of $\mathrm{THz}$ waves from a cluster plasma produced by double-pulse laser beams compared with that produced by a single-pulse beam. The angular distribution of the $\mathrm{THz}$ emission is then measured under conditions of a delay time of $133 \mathrm{ps}$ and $(x, y)=(0$, $40 \mu \mathrm{m})$. The propagation direction of the first laser pulse is denoted by $\Theta=0^{\circ}$. For a single-pulse beam, THz waves are emitted at $\pm 30^{\circ}$, which is consistent with previous results. ${ }^{11}$ For double-pulse laser beams, the THz power is increased by 

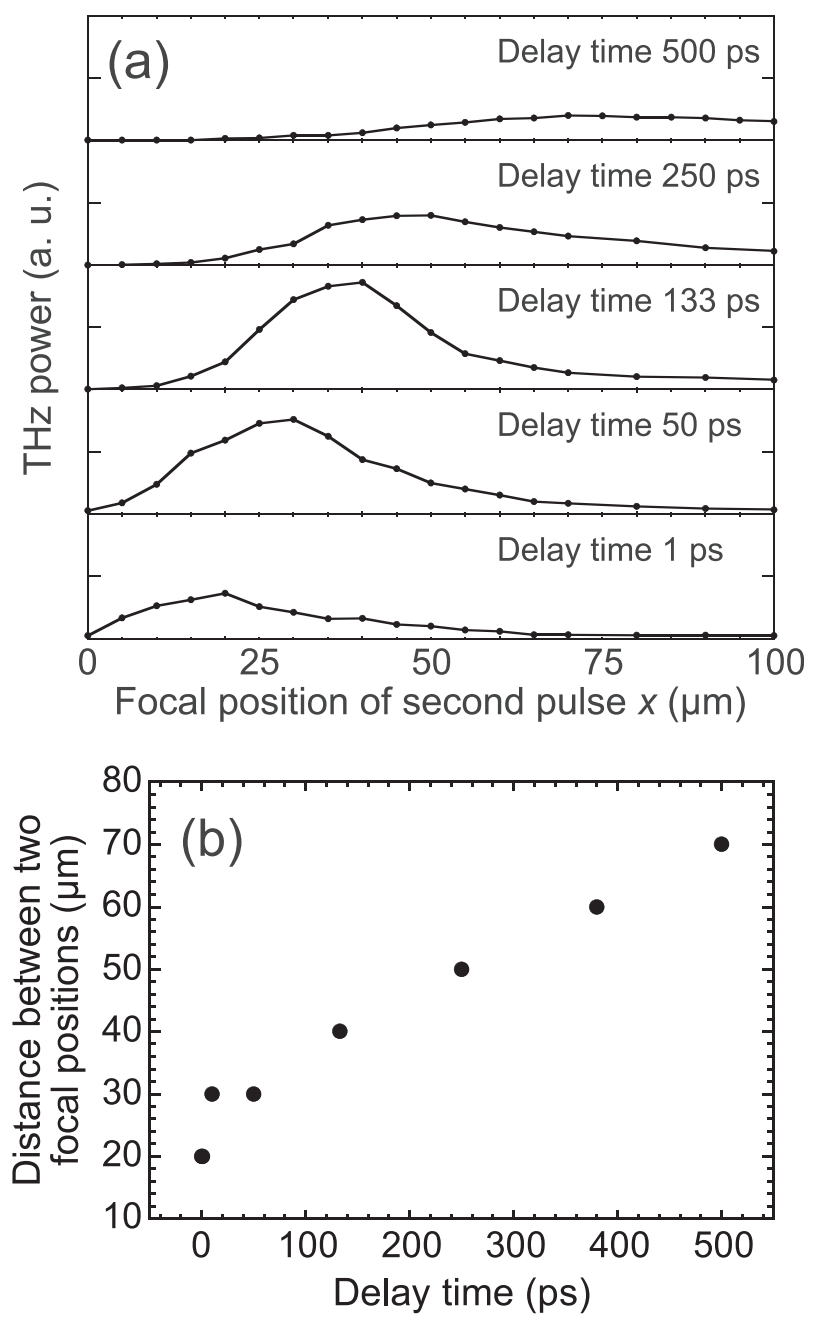

FIG. 3. (a) Temporal development of the THz power dependence with the distance between focal positions of the first and second pulses in the horizontal plane. (b) Distance between the two focal positions for various delay times at which the $\mathrm{THz}$ power reaches its peak value.

more than 10 times that for the single-pulse beam in the laser-propagation direction. The $\mathrm{THz}$ emission is directed much more in the laser-propagation direction when the first pulse forms the plasma and the second pulse generates the $\mathrm{THz}$ waves, whereas $\mathrm{THz}$ waves are emitted conically in the single-pulse case. The frequency of the $\mathrm{THz}$ waves is measured separately by the Martin-Puplett interferometer in the range of $0.03-2 \mathrm{THz}$. The observed spectrum of $\mathrm{THz}$ waves covers $0.1-1 \mathrm{THz}$ and peaks around $0.2-0.5 \mathrm{THz}$ with a frequency width (FWHM) of approximately $0.2-0.3 \mathrm{THz}$.

The polarization of the $\mathrm{THz}$ waves is measured using a wire grid. The experiment is performed at a laserpropagation direction of $\Theta=0^{\circ}$. The inset in Fig. 5(b) shows the transmitted $\mathrm{THz}$ wave power as a function of the wiregrid angle $\Phi$ for $\theta=-1^{\circ}$ and $57^{\circ}$. The $\mathrm{THz}$ waves are linearly polarized at every value of $\theta$. The polarization angle $\varphi$ of the $\mathrm{THz}$ waves is obtained by analyzing the wire-grid angle $\Phi$ for maximum transmission; the transmitted $\mathrm{THz}$ power is well fitted by the function $I=\cos ^{2}(\Phi-\varphi)$. The polarization angle of the $\mathrm{THz}$ waves is $4^{\circ}$ for $\theta=-1^{\circ}$ and $59^{\circ}$ for $\theta=57^{\circ}$ and increases with the second-pulse angle $\theta$. Figure 5(b) summarizes the polarization angle $\varphi$ of the $\mathrm{THz}$ waves for various angles $\theta$ of the second pulse, which shows

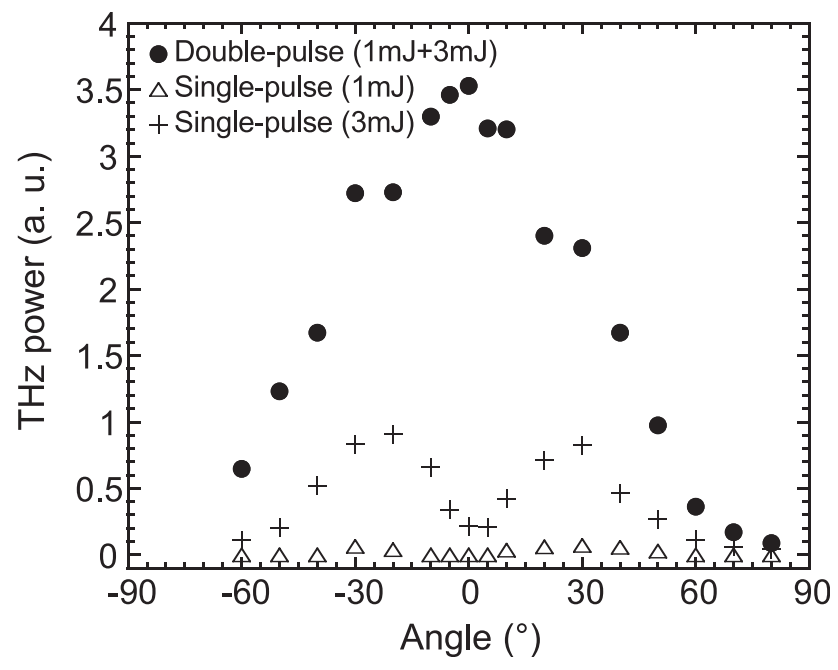

FIG. 4. Angular distributions of $\mathrm{THz}$ waves for a double-pulse beam (pulse energies of 1 and $3 \mathrm{~mJ}$ ) and a single-pulse beam (pulse energies of 1 or 3 $\mathrm{mJ}) ; 0^{\circ}$ is the laser-propagation direction. $\mathrm{THz}$ emission is measured under conditions of a delay time of $133 \mathrm{ps}$ and $(x, y)=(0,40 \mu \mathrm{m})$.

that $\varphi=\theta$. Thus, the polarization angle of linearly polarized $\mathrm{THz}$ waves can be rotated by the relative position of the first and second pulses without changing the incident laser polarization.

(a)
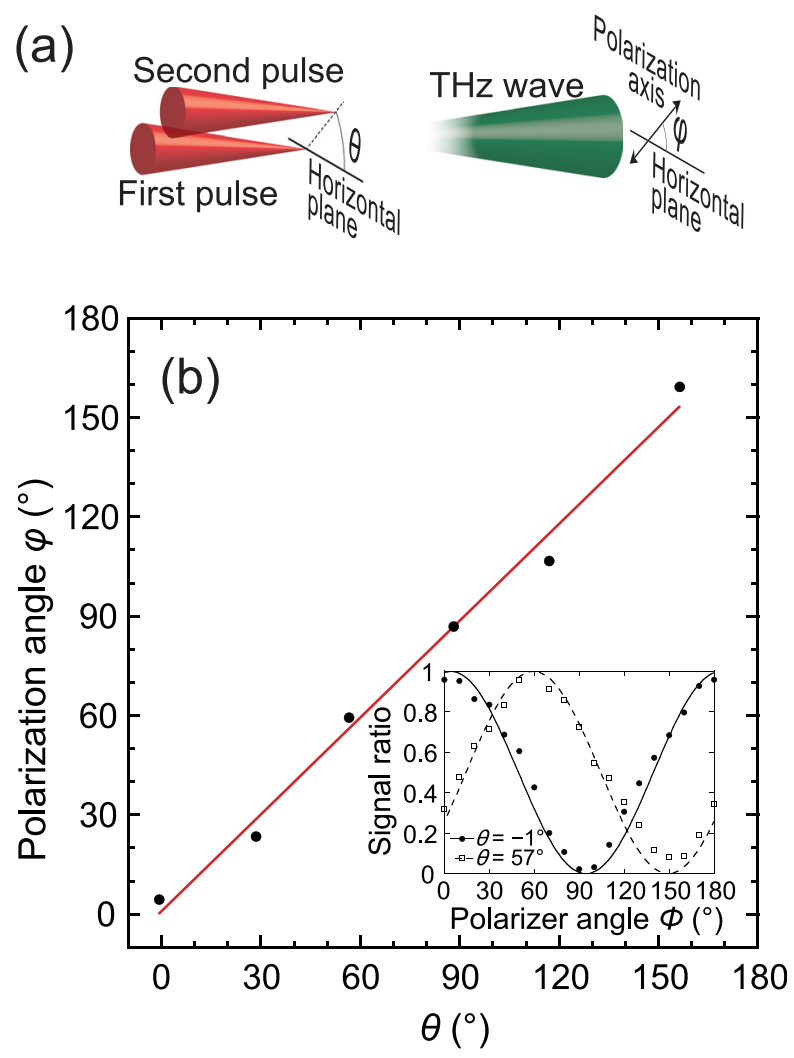

FIG. 5. (a) Schematic diagram of the definition of $\theta$ and $\varphi . \theta$ is defined as the angle between the line connecting the two focal positions and the horizontal plane. $\varphi$ is the angle between the direction of the vibration of the THz electric field and horizontal plane. (b) Polarization angle $\varphi$ of the $\mathrm{THz}$ wave for various $\theta$. The red line is the least-squares regression line $\varphi=0.97 \theta+1.0, \mathrm{R}=0.99$. The delay time between the two pulses is $133 \mathrm{ps}$. THz power is detected in the laser-propagation direction $\left(\Theta=0^{\circ}\right.$ in Fig. 1). Inset: $\mathrm{THz}$ transmission of the wire-grid polarizer for various wire-grid angles. Horizontal linearly polarized $\mathrm{THz}$ waves are transmitted by the wiregrid polarizer when the wire-grid angle is $0^{\circ}$. 
The characteristics of $\mathrm{THz}$ waves emitted from clusters irradiated by intense femtosecond double laser pulses can be summarized as follows: (1) higher directivity than for a single pulse, (2) high intensity enhancement with appropriate intervals between the double pulses in space and time, and (3) linear polarization in the direction of the line connecting the focal points of the double pulses. These characteristics are useful for applications because they result in (1) highly efficient use, (2) higher power, and (3) variable polarization, respectively.

Linearly polarized directional $\mathrm{THz}$ emission from plasmas cannot be explained by conventional mechanisms such as transition radiation ${ }^{22}$ or transition-Cherenkov radiation ${ }^{23}$ which have been studied as $\mathrm{THz}$ generation mechanisms for laser plasmas produced from gas targets. In the present work, because the laser intensity is in the range of $10^{16}-10^{17} \mathrm{~W} /$ $\mathrm{cm}^{2}$, it is expected that $\mathrm{THz}$ waves should be emitted in the direction $\Theta=80^{\circ}-90^{\circ} .{ }^{12}$ Although directional THz emission via four-wave optical rectification or second-order optical rectification inside an asymmetric filament ${ }^{24,25}$ and parametric decay of laser light ${ }^{26}$ have been reported in other works on $\mathrm{THz}$ generation using a plasma filament, the polarization of the $\mathrm{THz}$ waves was elliptical, not linear.

The generation of directional linearly polarized $\mathrm{THz}$ waves from clusters under double-pulse irradiation can be understood as the generation of $\mathrm{THz}$ waves from a plasma channel near Coulomb-exploded ions, which work as a bias. First, argon clusters are Coulomb-exploded by the first pulse in the focal space, and ions are emitted from the focal space to form expanding (positively charged) ion clouds. Then, the second pulse is irradiated from near the explosion front and provides electrons along the ion-cloud front as the second pulse travels. A transverse current ${ }^{27}$ or dipole moment ${ }^{28}$ would be excited in the plasma channel produced by the second pulse, resulting in emission of electromagnetic waves in the laser propagation direction just like $\mathrm{THz}$ wave generation in a DC field. ${ }^{27,29}$ The electromagnetic wave is emitted most efficiently at the ion-cloud front, which moves as fast as the ions that are emitted from the Coulomb-exploded clusters. Because the laser intensity of the first pulse is in the range of $10^{16}-10^{17} \mathrm{~W} / \mathrm{cm}^{2}$, the cluster survives as a skinned cluster. Based on the scaling law of the ionized radius with laser intensity, the remaining core part of the cluster is estimated to have a radius of $2.5 \mathrm{~nm}$. The maximum and probable energy of argon ions can be estimated as $E_{\max }=4 \pi Z^{2} e^{2} n_{\mathrm{i}}\left(R_{0}{ }^{3}-R^{3}\right) /$ $\left(3 R_{0}\right)$, where $Z$ is the ion charge state, $e$ is the electron charge, $n_{\mathrm{i}}$ is the ion density, and $R_{0}$ and $R$ are the radii of an initial argon cluster and remaining core argon cluster, respectively. For $R_{0}=5 \mathrm{~nm}, R=2.5 \mathrm{~nm}$, and $Z=7$, we have $E_{\max }=1.6$ $\times 10^{2} \mathrm{keV}$. However, both the laser intensity and cluster radius are distributed spatially, which contributes to the observed distributions in the actual experiments. We have reported previously that the most probable energy of argon ions is distributed in the energy range of $1-14 \mathrm{keV}$ at a laser intensity of $10^{16} \mathrm{~W} / \mathrm{cm}^{2} .{ }^{30}$ These energies correspond to speeds of $7 \times 10^{4}$ to $2.6 \times 10^{5} \mathrm{~m} / \mathrm{s}$, which agree well with the temporal development speed of the optimal distance of the focal positions in Fig. 3(b). In this mechanism, the $\mathrm{THz}$ wave is polarized in the direction along a line connecting the focal points of the double pulses and is consistent with the experimental results. The characteristics of the measured $\mathrm{THz}$ wave show that the combination of a cluster target and doublepulse irradiation is effective for generating $\mathrm{THz}$ waves. Future work will involve systematic studies of the mechanism of $\mathrm{THz}$ wave generation from cluster targets irradiated with doublepulse beams.

In summary, distinguishing properties have been observed in the emission of $\mathrm{THz}$ waves from argon clusters irradiated by intense femtosecond double laser pulses with appropriate intervals in time and space. These properties are high directivity, power enhancement, and linear polarization, which can be varied by changing the pointing of the second pulse relative to that of the first pulse. All these properties are useful for applications.

This work was financially supported by the Collaborative Research Program of the Institute for Chemical Research, Kyoto University (Grant Nos. 2016-4, 2017-2), and a Grantin-Aid for Scientific Research (C) (JP16K06745) from the Ministry of Education, Culture, Sports, Science and Technology (MEXT), Japan.

${ }^{1}$ X. C. Zhang, A. Shkurinov, and Y. Zhang, Nat. Photonics 11, 16 (2017).

${ }^{2}$ C. Kealhofer, W. Schneider, D. Ehberger, A. Ryabov, F. Krausz, and P. Baum, Science 352, 429 (2016).

${ }^{3}$ W. R. Huang, E. A. Nanni, K. Ravi, K. H. Hong, A. Fallahi, L. J. Wong, P. D. Keathley, L. E. Zapata, and F. X. Kärtner, Sci. Rep. 5, 14899 (2015).

${ }^{4}$ K. Kovács, E. Balogh, J. Hebling, V. Tosa, and K. Varjú, Phys. Rev. Lett. 108, 193903 (2012).

${ }^{5}$ M. Shalaby, C. Vicario, and C. P. Hauri, Appl. Phys. Lett. 108, 182903 (2016).

${ }^{6}$ C. Vicario, A. V. Ovchinnikov, S. I. Ashitkov, M. B. Agranat, V. E. Fortov, and C. P. Hauri, Opt. Lett. 39, 6632 (2014).

${ }^{7}$ A. Gopal, P. Singh, S. Herzer, A. Reinhard, A. Schmidt, U. Dillner, T. May, H.-G. Meyer, W. Ziegler, and G. G. Paulus, Opt. Lett. 38, 4705 (2013).

${ }^{8}$ T. Löffler, F. Jacob, and H. G. Roskos, Appl. Phys. Lett. 77, 453 (2000).

${ }^{9}$ C. D'Amico, A. Houard, M. Franco, B. Prade, A. Mysyrowicz, A. Couairon, and V. T. Tikhonchuk, Phys. Rev. Lett. 98, 235002 (2007).

${ }^{10}$ T. Nagashima, H. Hirayama, K. Shibuya, M. Hangyo, M. Hashida, S. Tokita, and S. Sakabe, Opt. Express 17, 8907 (2009).

${ }^{11}$ F. Jahangiri, M. Hashida, T. Nagashima, S. Tokita, M. Hangyo, and S. Sakabe, Appl. Phys. Lett. 99, 261503 (2011).

${ }^{12}$ F. Jahangiri, M. Hashida, S. Tokita, T. Nagashima, M. Hangyo, and S. Sakabe, Appl. Phys. Lett. 102, 191106 (2013).

${ }^{13}$ A. A. Frolov, Plasma Phys. Rep. 42, 637 (2016).

${ }^{14}$ A. V. Balakin, M. S. Dzhidzhoev, V. M. Gordienko, M. N. Esaulkov, I. A. Zhvaniya, K. A. Ivanov, I. A. Kotelnikov, N. A. Kuzechkin, I. A. Ozheredov, V. Y. Panchenko, A. B. Savel'ev, M. B. Smirnov, P. M. Solyankin, and A. P. Shkurinov, IEEE Trans. Terahertz Sci. Technol. 7, 70 (2017).

${ }^{15}$ D. J. Cook and R. M. Hochstrasser, Opt. Lett. 25, 1210 (2000).

${ }^{16}$ J. Das and M. Yamaguchi, J. Opt. Soc. Am. B 30, 1595 (2013).

${ }^{17}$ R. G. Ungureanu, O. V. Grigore, M. P. Dinca, G. V. Cojocaru, D. Ursescu, and T. Dascalu, Laser Phys. Lett. 12, 045301 (2015).

${ }^{18}$ X. Xie, J. Dai, and X.-C. Zhang, Phys. Rev. Lett. 96, 075005 (2006).

${ }^{19}$ Y. Liu, A. Houard, B. Prade, S. Akturk, A. Mysyrowicz, and V. T. Tikhonchuk, Phys. Rev. Lett. 99, 135002 (2007).

${ }^{20}$ M. Hirokane, S. Shimizu, M. Hashida, S. Okada, S. Okihara, F. Sato, T. Iida, and S. Sakabe, Phys. Rev. A 69, 063201 (2004).

${ }^{21}$ M. Naftaly and R. E. Miles, J. Non-Cryst. Solids 351, 3341 (2005).

${ }^{22}$ U. Happek, A. J. Sievers, and E. B. Blum, Phys. Rev. Lett. 67, 2962 (1991).

${ }^{23}$ C. D'Amico, A. Houard, S. Akturk, Y. Liu, J. L. Bloas, M. Franco, B. Prade, A. Couairon, V. T. Tikhonchuk, and A. Mysyrowicz, New J. Phys. 10, 013015 (2008).

${ }^{24}$ Y. Chen, C. Marceau, W. Liu, Z.-D. Sun, Y. Zhang, F. Théberge, M. Châteauneuf, J. Dubois, and S. L. Chin, Appl. Phys. Lett. 93, 231116 (2008). 
${ }^{25}$ Y. Zhang, Y. Chen, C. Marceau, W. Liu, Z.-D. Sun, S. Xu, F. Théberge, M. Châteauneuf, J. Dubois, and S. L. Chin, Opt. Express 16, 15483 (2008).

${ }^{26}$ F. Jahangiri, M. Hashida, S. Tokita, T. Nagashima, K. Otani, M. Hangyo, and S. Sakabe, Appl. Phys. Express 5, 026201 (2012).

${ }^{27}$ A. Houard, Y. Liu, B. Prade, V. T. Tikhonchuk, and A. Mysyrowicz, Phys. Rev. Lett. 100, 255006 (2008).
${ }^{28}$ N. A. Panov, O. G. Kosareva, V. A. Andreeva, A. B. Savel'ev, D. S. Uryupina, R. V. Volkov, V. A. Makarov, and A. P. Shkurinov, JETP Lett. 93, 638 (2011).

${ }^{29}$ T. Loffler, M. Kress, M. Thomson, and H. G. Roskos, Acta Phys. Pol. A 107, 99 (2005).

${ }^{30}$ S. Sakabe, K. Shirai, M. Hashida, S. Shimizu, and S. Masuno, Phys. Rev. A 74, 043205 (2006). 